\title{
Enhanced Ferromagnetism in ZnO Nanoribbons and Clusters Passivated with Sulfur
}

\author{
Andrés R. Botello-Méndez, Florentino López-Urías, Mauricio Terrones $(\bowtie)$, and Humberto Terrones \\ National Laboratory for Nanoscience and Nanotechnology Research (LINAN), Advanced Materials Department, IPICYT, Camino a la \\ Presa San José 2055, 78216, S. L. P., México \\ Received: 28 August 2008/ Revised: 19 September 2008/ Accepted: 23 September 2008 \\ CTsinghua Press and Springer-Verlag 2008. This article is published with open access at Springerlink.com
}

\begin{abstract}
Inspired by recent experimental results, the electronic and magnetic properties of sulfur-passivated $\mathrm{ZnO}$ clusters and zigzag nanoribbons have been studied using first principles calculations in the framework of the local spin density approximation. In the case of the $\mathrm{ZnO}$ nanoribbons, the sulfur atoms or thiol groups were attached in different ways to the zinc or oxygen atoms located at the edges, whereas in clusters, the sulfur atoms were set on the surface, mainly interacting with atoms with low-coordinate number. After an exhaustive atomic relaxation, we found that a magnetic moment emerges in zigzag nanoribbons both with and without sulfur-passivation on the edges. However, the magnitude of the magnetic moment is very sensitive to sulfur passivation. In particular, we found that when sulfur is attached to the zinc atoms in an alternating fashion along the ribbon edges, the magnetic moment is a maximum $\left(1.4 \mu_{\mathrm{B}} /\right.$ unit cell). In the case of clusters, we found that the $\mathrm{Zn}_{15} \mathrm{O}_{15}$ cluster exhibits a high spin moment of $5.5 \mu_{\mathrm{B}}$ when capped with sulfur atoms. Our calculations indicate that sulfur-passivating of $\mathrm{ZnO}$ nanosystems could be responsible for recently observed ferromagnetic responses.
\end{abstract}

\section{KEYWORDS}

Zinc oxide, sulfur, magnetism, nanoribbons, clusters

\section{Introduction}

Metal oxide nanostructures have been recently investigated from both experimental and theoretical standpoints due to their potential applications in the fabrication of novel electronic devices. In this context, one of the most versatile systems is zinc oxide $(\mathrm{ZnO})$. In particular, for spintronics, $\mathrm{ZnO}$ doped with transition metals has been predicted to be one of the best candidates [1]. The recent observation of ferromagnetism in undoped semiconductor oxide nanoparticles $\left(\mathrm{TiO}_{2}, \mathrm{ZnO}, \mathrm{In}_{2} \mathrm{O}_{3}, \mathrm{SnO}_{2}\right.$, and $\left.\mathrm{CeO}_{2}\right)$ [2] and other unusual magnetic responses in $\mathrm{ZnO}$ doped with transition metals [3, 4], have created excitement in order to answer questions regarding the origin of magnetism in these systems. García et al. have found a magnetic behavior in $\mathrm{ZnO}$ nanoparticles with organic passivation [5]. They reported that nanoparticles grown in a dodecanethiol environment present a large ferromagnetic response. Banerjee et al. observed an enhancement of ferromagnetism upon thermal annealing of $\mathrm{ZnO}$ nanoparticles grown by

Address correspondence to mterrones@ipicyt.edu.mx 
a micellar method from a sulfur-based compound [6]. Recent calculations using the local spin density approximation (LSDA) showed that ferromagnetism could be found in pure $\mathrm{ZnO}$ or in carbon-doped $\mathrm{ZnO}$ clusters [7]. Despite the fact that doping and/ or passivating $\mathrm{ZnO}$ with non-magnetic ions clearly results in magnetic properties, the origin of this unusual effect is far from clear.

It is the aim of this manuscript to shed some light on the effect of sulfur atoms on the magnetic properties of $\mathrm{ZnO}$ systems using first principles calculations. We demonstrate that sulfur-passivation in $\mathrm{ZnO}$ systems promotes strong ferromagnetism. This finding is significant due to its possible technological applications in information storage, processing devices and spintronics. Using density functional theory, we predict ferromagnetism enhancement in two $\mathrm{ZnO}$ systems-nanoparticles and nanoribbons-when passivated with sulfur or thiol groups. Recent experimental results, related to the production of very thin films of $\mathrm{ZnO}$ adopting a boron-nitride-like planar structure, have inspired our choice for one of the systems studied here [8]. Recently, we have observed using first principles calculations that one-dimensional undoped $\mathrm{ZnO}$ nanostructures, and in particular zigzag nanoribbons, are metallic and can exhibit small ferromagnetic responses $[9,10]$. When these ribbons are passivated with sulfur or methanethiol $\left(\mathrm{CH}_{3} \mathrm{SH}\right)$, however, the magnetic moment increases significantly. Local densities of states (LDOS) calculated on the sulfur, zinc, and oxygen atoms within the $\mathrm{ZnO}$ nanoribbon unit cell indicate that the main contribution to the total magnetic moment arises from the sulfur atoms. Similarly, we have also found that $\mathrm{ZnO}$ clusters can exhibit enhanced ferromagnetism when they are passivated with sulfur atoms and a high magnetic moment is obtained. The ferromagnetic behavior found in these sulfur-passivated $\mathrm{ZnO}$ nanoparticles is attributed to weakly coordinated atoms located on the cluster surface.

\section{Results and discussion}

In Fig. 1, we show five different forms of sulfurpassivated $\mathrm{ZnO}$ zigzag nanoribbons that were constructed by cutting a planar monolayer along two parallel zigzag lines. The ribbons are periodic in the " $x$ " direction with the width defined in the " $y$ " direction. Note that if the ribbons were periodic



(a) $S_{z n, 0}$

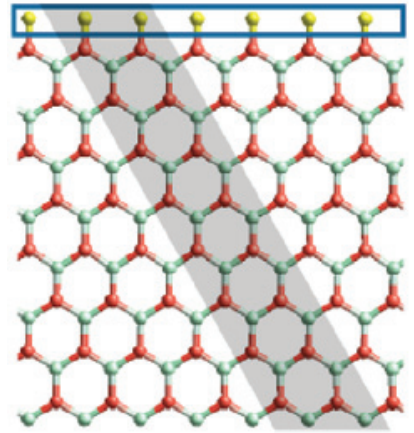

(b) $\mathrm{S}_{0}$

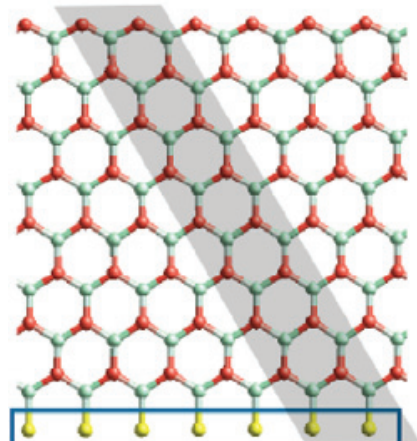

(c) $\mathrm{S}_{\mathrm{zn}}$
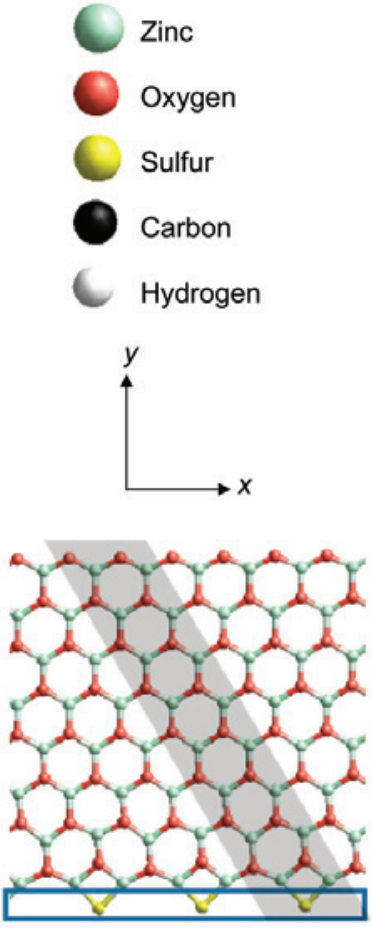



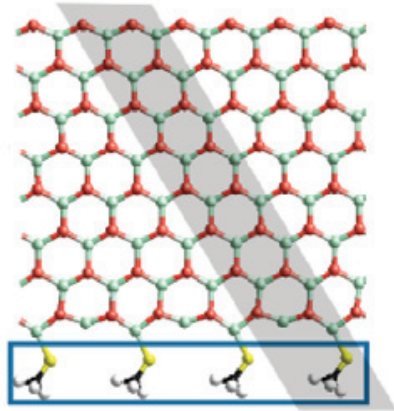

(e) $S_{\text {Thiol }}$
Figure 1 Molecular models of $\mathrm{ZnO}$ single-layered nanoribbons passivated with sulfur along the edges (the ribbons are periodic in the horizontal direction). In all systems, the sulfur passivation is denoted by a rectangle, and the shaded region represents a unit cell: (a) both the $\mathrm{Zn}$ - and the O-dominated edges are passivated; (b) only the O-dominated edge is passivated; (c) only the $\mathrm{Zn}$-dominated edge is passivated; (d) alternating sulfur passivation of the $\mathrm{Zn}$-dominated edge; (e) a thiol is attached to a $\mathrm{Zn}$ atom. All the structures were relaxed using a conjugate gradient method. It is important to remark that all ribbons exhibit a magnetic moment. In case (d), where the sulfur atoms are attached to two zinc atoms, the magnetic moment is maximal 
in the " $y$ " direction, they would exhibit armchair edges. However, we are not interested in this kind of ribbons since they are semiconducting [9] and do not exhibit ferromagnetic behavior [10]. For all zigzag nanoribbons, the sulfur or thiol groups are attached to the ribbon atoms located on the edges. The shaded regions in Fig. 1 schematically represent the unit cell; for details see the Methods section below.

Previous electronic calculations carried out for a few number of $\mathrm{ZnO}$ layers have demonstrated that after relaxation, the structure prefers a planar configuration in which both the cation $(\mathrm{Zn})$ and the anion $(\mathrm{O})$ share the same plane [11]. These predictions were recently confirmed experimentally by Tusche et al. by depositing $\mathrm{ZnO}$ on Ag(111) using laser ablation [8]. Therefore, all the structures considered in this work were constructed from planar layers, and subsequently minimized energetically. Recently, we have also demonstrated that undoped or unpassivated $\mathrm{ZnO}$ zigzag nanoribbons formed by a single layer are metallic and can exhibit a small magnetic moment [10].

We first studied nanoribbons passivated with sulfur on both edges (labelled $S_{\mathrm{Zn}, \mathrm{O}}$, see Fig. 1(a)). Here, a sulfur atom is attached to a zinc or oxygen atom. The spin-resolved density of states (Fig. 2(a)) shows a shift of the spin-up and spin-down densities with the magnetic moment $\left(0.59 \mu_{\mathrm{B}}\right)$ being approximately $10 \%$ lower than that of the corresponding unpassivated system $\left(0.65 \mu_{\mathrm{B}}\right)$. We have previously shown that the main contribution to the total magnetic moment comes from the oxygendominated edge [10]. Therefore, the introduction of sulfur-passivation on both edges does not significantly affect the electronic and magnetic properties of the nanoribbons. However, the magnetic moment of the nanoribbons varies depending on how the sulfur atoms are attached to the edges (see below).

Figures 1(b) and 1(c) depict the morphology of the $\mathrm{ZnO}$ zigzag nanoribbons passivated on the oxygenterminated edge (labelled $\mathrm{S}_{\mathrm{O}}$ ), and zinc-terminated edge (labelled $\mathrm{S}_{\mathrm{Zn}}$ ), respectively. In both cases, the spin resolved density of states reveals a shift in the up and down spins, although it is substantially less in the former case. The magnetic moment of the $S_{\mathrm{O}}$ ribbon $\left(0.12 \mu_{\mathrm{B}}\right)$ is only $18 \%$ of the magnetic moment of the unpassivated $\mathrm{ZnO}$ nanoribbon, while the magnetic moment of the $\mathrm{S}_{\mathrm{Zn}}$ ribbon $\left(0.72 \mu_{\mathrm{B}}\right)$ is increased by approximately $10 \%$. In addition, the

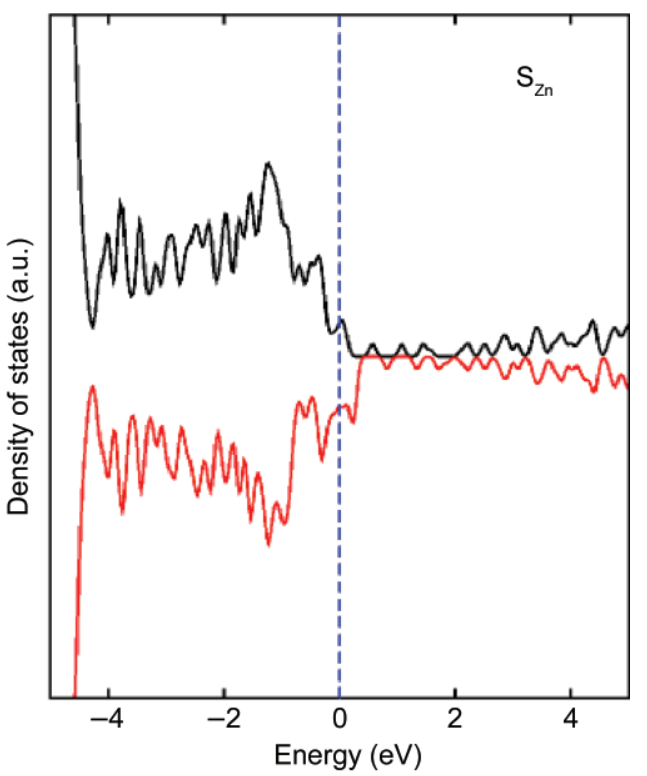

(a)

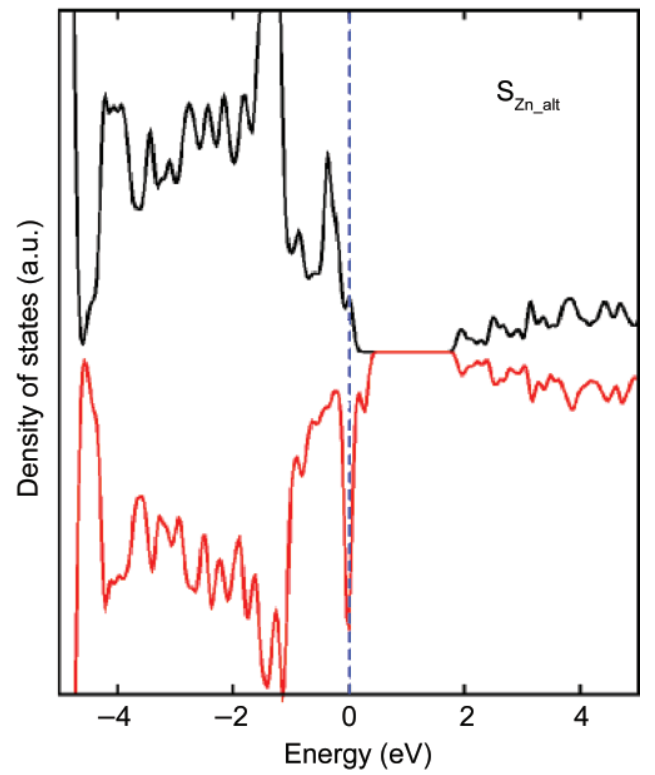

(b)

Figure 2 Spin-polarized electronic density of states of $\mathrm{ZnO}$ nanoribbons passivated with sulfur (note that all systems show a shift in the density of states, and consequently a magnetic moment is obtained): (a) nanoribbon passivated on the $\mathrm{Zn}$-terminated edge showing $\mu=0.72 \mu_{\mathrm{B}}$ (the structure can be seen in Fig. 1(c)); (b) nanoribbon passivated on the Zn-terminated edge in an alternating form, showing an increased magnetic moment of $1.4 \mu_{\mathrm{B}}$ (the structure can be seen in Fig. 1(d)). The magnetic moment of the unpassivated ribbon is $0.65 \mu_{\mathrm{B}}$ 
$\mathrm{S}_{\mathrm{Zn}}$ ribbon is more energetically stable than the $\mathrm{S}_{\mathrm{O}}$ ribbon, as $\mathrm{S}$ tends to bind preferentially to $\mathrm{Zn}$ instead of $\mathrm{O}$.

From the experimental point of view, it is difficult to have sulfur passivating both of the two coordinate atoms (oxygen and zinc) within the nanoribbon. Moreover, sulfur has several oxidation states. Therefore, we tried to passivate the zinc-dominated edge with a single sulfur atom every two $\mathrm{Zn}$ atoms (alternating passivation), labelled $S_{Z n \_a l t}$ in Fig. 1(d). The $S_{\text {Zn_alt }}$ nanoribbon still exhibits states at the Fermi level, and a shift in the DOS, as shown in Fig. 2(b). Here, the magnetic moment $\left(1.4 \mu_{\mathrm{B}}\right)$ is more than twice that of the unpassivated ribbon $\left(0.65 \mu_{\mathrm{B}}\right)$.

Figure 3 depicts the LDOS for the $\mathrm{S}_{\mathrm{Zn} \_ \text {alt }}$ nanoribbon. The DOS was calculated in all the zinc sites, and then added. A similar procedure was followed for the case of sulfur and oxygen. It can be observed that all types of atoms ( $\mathrm{Zn}, \mathrm{O}$, and S) exhibit states at the Fermi level, and a shift in the spin-up and spin-down densities. For sulfur, only states with spin-down can

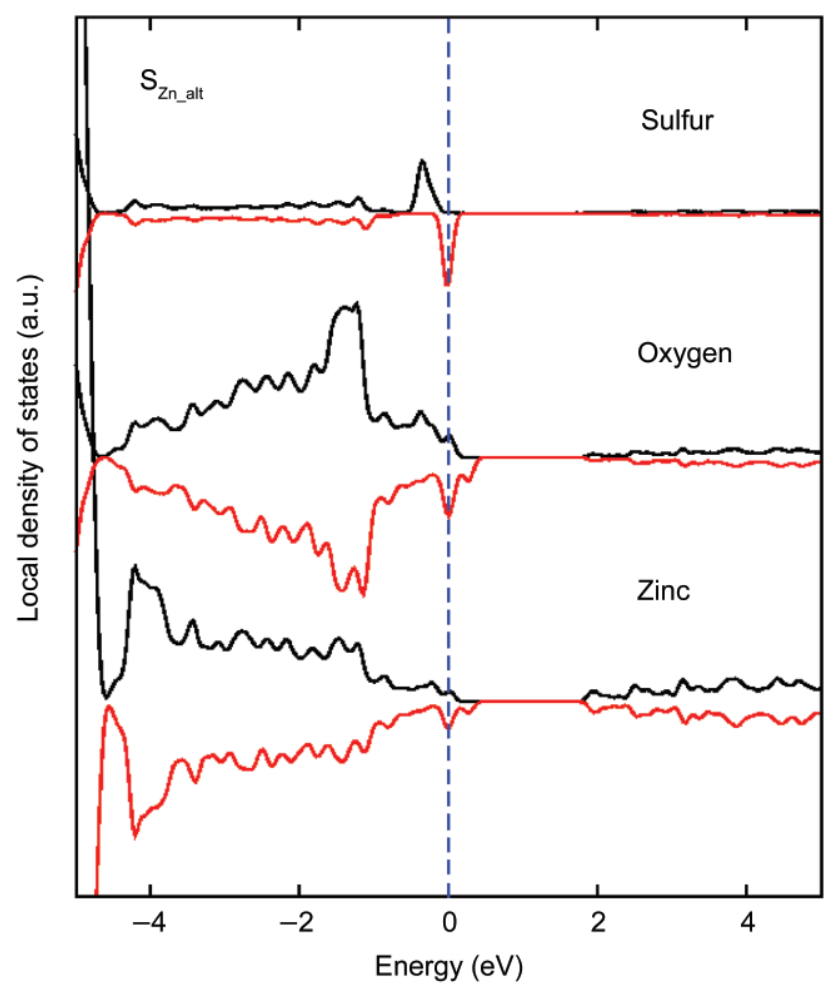

Figure 3 Spin-polarized local electronic density of states for the nanoribbons with alternating $S$ atoms along to the $\mathrm{Zn}$-terminated edges (the structure can be seen in Fig. 1(d)). Results for sulfur, zinc, and oxygen atoms are shown. Notice that all atoms exhibit a shift between the spin-up and spin-down density of states, and consequently all atoms contribute to the total magnetic moment be observed at the Fermi level with almost zero states in the conduction band, contrary to the situation for oxygen and zinc atoms. From magnetic moment calculations, we note that the sulfur atoms make a major contribution to the total magnetic moment.

Additionally, the effect of passivating a $\mathrm{ZnO}$ zigzag nanoribbon with a thiol moiety was investigated. In this case, a methanethiol $\left(\mathrm{CH}_{3} \mathrm{SH}\right)$ group was attached to two $\mathrm{Zn}$ atoms on the $\mathrm{Zn}$ dominated edge of the zigzag nanoribbons (Fig. 1(e); labelled $S_{\text {Thiol }}$ ). After geometric optimization, the sulfur atom is not bound to two $\mathrm{Zn}$ atoms. The DOS of this system (not shown here) displays a shift in the spin-up and spin-down densities, and exhibits a magnetic moment of approximately $1 \mu_{\mathrm{B}}$. Since the calculations of the ribbons with the thiol moieties are very expensive in terms of computational time, we only performed one case. However, it is clear from this exercise that the effect of passivating with thiol groups is qualitatively the same as for pure sulfur passivation.

García et al. demonstrated that thiol passivated nanoparticles exhibit a ferromagnetic response [5]. Therefore, we also investigated the effect of passivating a $\mathrm{ZnO}$ nanocluster with sulfur atoms. We performed spin-polarized calculations on a $\mathrm{Zn}_{15} \mathrm{O}_{15}$ cluster constructed from the wurtzite crystal, and attached sulfur atoms to each of the $\mathrm{Zn}$ atoms with coordination number less than four (see the unrelaxed cluster in Fig. 4). The whole structure was then energetically minimized. In this case, although the unpassivated $\mathrm{Zn}_{15} \mathrm{O}_{15}$ showed a zero magnetic moment without states at the Fermi level [7], the sulfur-passivated cluster exhibits a huge magnetic moment $\left(\mu=5.5 \mu_{\mathrm{B}}\right)$ and states at the Fermi level. As in the case of the nanoribbons, the magnetic moment arises mainly from the sulfur atoms. We note from the relaxed molecule that the sulfur atoms tend to bond with other sulfur atoms and with $\mathrm{Zn}$ atoms rather than with oxygen atoms. This observation is consistent with the nanoribbon case in which the $S_{Z n}$ is more stable than $S_{O}$. The results for this particular cluster clearly confirm that sulfur atoms play a crucial role in determining the ferromagnetic properties. The DOS of the $\mathrm{Zn}_{15} \mathrm{O}_{15}$ cluster is interesting because it exhibits only spin-

\section{量 Springer}




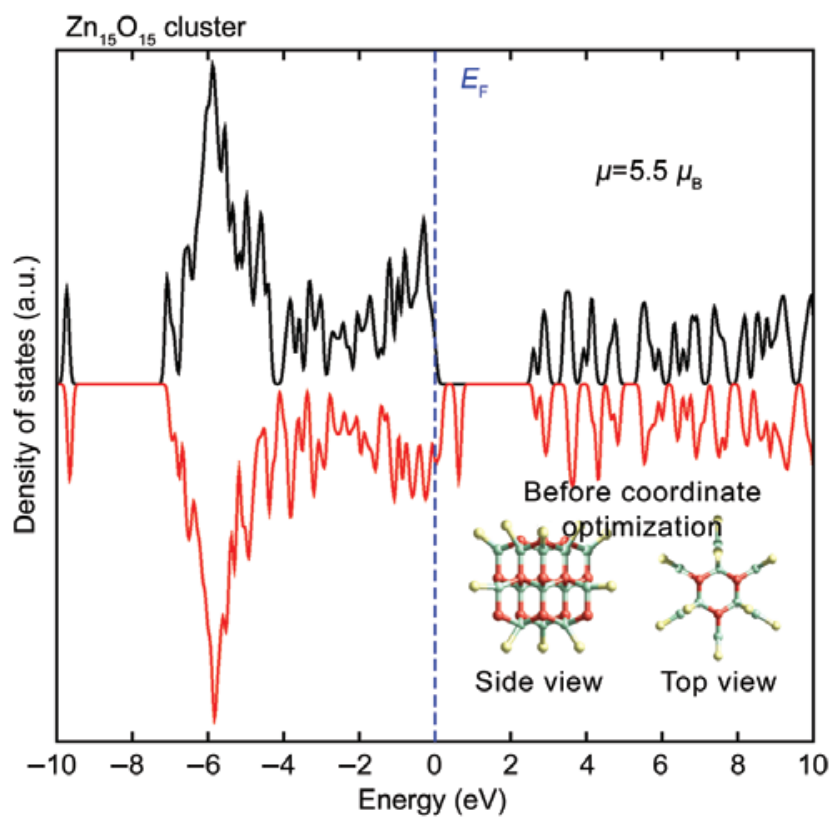

Figure 4 Spin-polarized electronic density of states of the $\mathrm{Zn}_{15} \mathrm{O}_{15}$ cluster passivated with sulfur on the surface. Here, the $S$ atoms are attached to $\mathrm{Zn}$ atoms with low coordination number (2 or 3 nearest neighbors). The molecular structure can be seen in the inset. The Fermi level is set to zero. The cluster exhibits high spin moment $\left(5.5 \mu_{\mathrm{B}}\right)$. The structure was relaxed using the conjugate gradient method. It is important to remark that the pure $\mathrm{ZnO}$ cluster exhibits a null magnetic moment

down excitation states. This kind of material would be very interesting for spintronic applications, since a spin coherent excitation could be used for different channels of electronic transport.

Certainly, the idea of magnetism in systems containing non magnetic atoms (or $\mathrm{d}^{0}$ ferromagnetism), challenges our understanding because exchange mechanisms cannot be responsible for this behavior. Coey has reviewed different systems exhibiting $\mathrm{d}^{0}$ ferromagnetism, and he has proposed that this behavior can be induced by lattice defects and impurities giving rise to magnetic moments associated with molecular orbitals localized in the vicinity of the defects [12]. In the systems studied here, the major components of the magnetic moment are $\mathrm{p}$ orbital magnetic moments (shown in Fig. 5) of the atoms localized in the vicinity of the sulfur impurities. The contribution to the total DOS of the p orbitals is the sum of the oxygen $2 p$, sulfur $3 p$, and zinc $4 p$ orbitals. These orbitals also make the major contribution to the conduction states.

\section{Conclusions}

We have demonstrated the importance of sulfur- and thiol-passivation in $\mathrm{ZnO}$ nanoribbons and clusters. We believe that our results can explain the recent experimental observations of ferromagnetism observed in $\mathrm{ZnO}$ nanoparticles, because the presence of sulfur plays a crucial role in determining the magnetic properties of the different systems investigated in this work. For example, a pure $\mathrm{Zn}_{15} \mathrm{O}_{15}$ cluster exhibits a null magnetic moment, whereas if the same cluster is passivated with sulfur atoms, a large magnetic moment is obtained $\left(5.5 \mu_{\mathrm{B}}\right)$. In addition, zigzag nanoribbons are excellent candidates for ferromagnetic materials. We found that when the sulfur atoms are attached to $\mathrm{Zn}$ atoms in an alternating way along one edge, the magnetic moment is increased significantly. In summary, the findings related to ferromagnetism in nanostructures doped with non-magnetic elements are novel and interesting due to the potential applications of these new magnetic materials in the data storage industry.

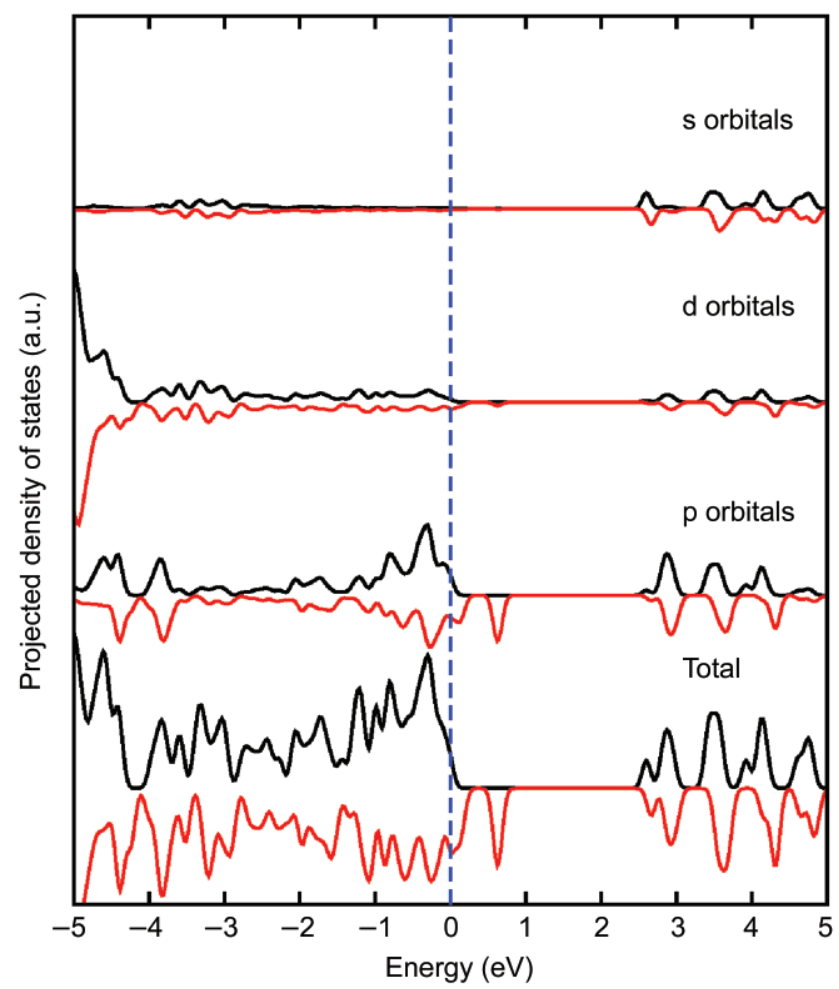

Figure 5 Projected density of states of the $\mathrm{Zn}_{15} \mathrm{O}_{15}$ cluster passivated with sulfur. The contribution of the $p$ orbitals to the magnetic moment shows that the magnetism of these systems cannot be attributed to the transition metal, but rather to an orbital magnetism 


\section{Methods}

The electronic calculations were carried out using density functional theory (DFT) in the framework of the LSDA with a basis of linear combination of atomic orbitals (LCAO) as implemented in the SIESTA code [13]. We use a double- $\zeta$ basis set with additional polarization orbitals. The pseudo-potentials (pps) were constructed from 12, 6, and 6 valence electrons for the $\mathrm{Zn}, \mathrm{O}$, and $\mathrm{S}$ atoms, respectively $\left(\mathrm{Zn}: 4 \mathrm{~s}^{2} 3 \mathrm{~d}^{10}\right.$, O: $\left.2 s^{2} 2 p^{4}, s: 3 s^{2} 3 p^{4}\right)$. The Troullier-Martins scheme was used. The basis set and the pps were tested on the bulk zinc oxide wurtzite structure, which showed an energy gap $\left(E_{\mathrm{g}}\right)$ of $0.76 \mathrm{eV}$, and cell parameters consistent with previous local density approximation (LDA) calculations. No spin polarization was observed for the bulk wurtzite case.

It has been previously reported that the use of a generalized gradient approximation (GGA) functional rather than a local density approximation (LDA) underestimates the electronic band gap even more, and overestimates the cell parameters $(1.6 \%$ for GGA as compared to $-0.8 \%$ for LDA for the cell parameter c) [14]. In our previous work, we also verified that the approximations used in our work do not give artificial metallicity or spin polarization, using a plane wave basis and hybrid functionals. In this work, we also verified that by neglecting the electron-electron interaction when using LDAs, the results presented here do not change significantly and the ferromagnetic effects do indeed occur.

In this context, we performed DFT calculations within the $\mathrm{LDA}+U$ approximation using a plane wave basis as implemented in the Quantum-ESPRESSO package [15]. The energy cut-off of the plane waves was set to $65 \mathrm{Ry}$ and $8 \mathrm{~K}$-points were used for the sampling of the Brillouin zone. The determination of the Hubbard $U$ parameter for ab initio calculations is rather complicated, and several approaches have been used. Here, we adopted the proposal of Janotti et al. [16], where the $U$ parameter is defined as the energy interaction between the $\mathrm{d}$ electrons on the same atom screened by the macroscopic optical dielectric constant. For a nanoribbon or monolayer, the screening is very different from the bulk. Therefore, using the same $U$ as in the bulk would not be a very accurate approximation. In order to address this issue, we calculated the dielectric constant for a monolayer, which is a better estimate for the dielectric constant of the nanoribbons. The resulting value for $U$ was $11.6 \mathrm{eV}$. Using this effective on-site Coulomb correlation interaction, we found that the correction introduced by $\mathrm{LDA}+U$ changes the position of the $d$ bands, shifting them several $\mathrm{eV}$ below the valence band maximum, leading to a decreased interaction with the $\mathrm{O} p$ bands. Despite the difference in the lower valence bands, the description of the LDA method is qualitatively the same and correct, since the LDA $+U$ result exhibits states at the Fermi level, and a magnetic moment of $0.25 \mu_{\mathrm{B}}$ for the unpassivated zigzag nanoribbon.

All the nanoribbon structures in this work were constructed from planar $\mathrm{ZnO}$, and calculations were carried out on large periodic unit cells that prevent unrealistic interactions. The sulfur atoms were attached in different fashions, and the whole structures were further relaxed using a conjugate gradient (CG) algorithm with variable cell. The $\mathrm{Zn}_{15} \mathrm{O}_{15}$ cluster was constructed from the wurtzite structure, and the sulfur atoms were bound to the surface, and then minimized using CG with a force threshold of $0.04 \mathrm{eV} / \mathrm{A}$ which assures the structure is at least close (within the threshold) to a local minimum structure.

\section{Acknowledgements}

The authors are grateful to D. Ramírez, G. Ramírez, and G. Pérez for technical assistance. This work was supported in part by CONACYT-México grants: 56787 (Laboratory for Nanoscience and Nanotechnology Research-LINAN), 60218 (FLU), 45762(HT), 45772 (MT), 58899-Inter American Collaboration (MT), 2004-01-013/SALUD-CONACYT (MT), Fondo Mixto de San Luis Potosí 63001 S-3908 (MT), Fondo Mixto de San Luis Potosí 63072 S-3909 (HT), and a PhD Scholarship (ARBM).

\section{References}

[1] Dietl, T.; Ohno, H.; Matsukura, F.; Cibert, J.; Ferrand, D. Zener model description of ferromagnetism in zinc 
blende magnetic semiconductors. Science 2000, 287, 1019-1022.

[2] Sundaresan, A.; Bhargavi, R.; Rangarajan, N.; Siddesh, U.; Rao, C. N. R. Ferromagnetism as a universal feature of nanoparticles of the otherwise nonmagnetic oxides. Phys. Rev. B 2006 74, 161306.

[3] Ronning, C.; Gao, P. X.; Ding, Y.; Wang, Z. L.; Schwen, D. Manganese-doped $\mathrm{ZnO}$ nanobelts for spintronics. Appl. Phys. Lett. 2004, 84, 783-785.

[4] Rao, C. N. R.; Deepak, F. L. Absence of ferromagnetism in $\mathrm{Mn}$ - and Co- doped ZnO. J. Mater. Chem. 2005, 15, 573-578.

[5] Garcia, M. A.; Merino, J. M.; Fernández Pinel, E.; Quesada, A.; de la Venta, J.; Ruíz González, M. L.; Castro, G. R.; Crespo, P.; Llopis, J.; González Calbet, J. M.; Hernando, A. Magnetic properties of $\mathrm{ZnO}$ nanoparticles. Nano Lett. 2007, 7, 1489-1494.

[6] Banerjee, S.; Mandal, M.; Gayathri, N.; Sardar, M. Enhancement of ferromagnetism upon thermal annealing in pure ZnO. Appl. Phys. Lett. 2007, 91, 182501.

[7] Botello-Méndez, A. R.; López Urías, F.; Terrones, M.; Terrones, H. Unpublished results.

[8] Tusche, C.; Meyerheim, H. L.; Kirschner, J. Observation of depolarized ZnO (0001) monolayers: Formation of unreconstructed planar sheets. Phys. Rev. Lett. 2007, 99, 026102.

[9] Botello-Méndez, A. R.; López Urías, F.; Terrones, M.;
Terrones, $\mathrm{H}$. Magnetic behavior in zinc oxide zigzag nanoribbons. Nano Lett. 2008, 8, 1562-1565.

[10] Botello-Méndez, A. R.; Martínez-Martínez, M. T.; López Urías, F.; Terrones, M.; Terrones, H. Metallic edges in zinc oxide nanoribbons. Chem. Phys. Lett. 2007, 448, 258263.

[11] Claeyssens, F.; Freeman, C. L.; Allan, N. L.; Sun, Y.; Ashfold, M. N. R.; Harding, J. H. Growth of ZnO thin films-Experiment and theory. J. Mater. Chem. 2005, 15, 139-148.

[12] Coey, J. M. D. d ferromagnetism. Solid State Sci. 2005, 7, 660-667.

[13] Soler, J. M.; Artacho, E.; Gale, J. D.; García, A.; Junquera, J.; Ordejón, P.; Sánchez-Portal, D. The SIESTA method for ab initio order-N materials simulation. J. Phys. Condens. Matter. 2002, 14, 2745-2779.

[14] Meyer, B.; Marx, D. Density-functional study of the structure and stability of ZnO surfaces. Phys. Rev. B 2003 $67,035403$.

[15] Quantum-ESPRESSO is a community project for high quality quantum-simulation software, based on density functional theory, and coordinated by Paolo Giannozzi. See http://www.quantum-espresso.org and http://www. pwscf.org (Accessed 4 March, 2008).

[16] Janotti, A.; Segev, D.; Van de Walle, C. G. Effects of cation $d$ status on the structural and electronic properties of III-nitride and II-oxide wide-band-gap semiconductors. Phys. Rev. B 2006, 74, 045202. 